Revista Científica de FAREM-Estelí

Medio ambiente. tecnología y desarrollo humano

Año 10 | Edición especial: artículos de revisión documental

ISSN: 2305-5790

https://rcientificaesteli.unan.edu.ni

DOI: https://doi.org/10.5377/farem.v0i0.11617

\section{Eficiencia energética: una tarea para las universidades}

\section{Energy efficiency: a task for universities}

\section{Silvia Elena Arróliga Galeano}

Estudiante de doctorado en Gestión y Calidad de la Investigación Científica II Cohorte 2020-2023 (UNAN-Managua). Docente de la Universidad Nacional

Autónoma de Nicaragua, FAREM-Estelí.

https://orcid.org/0000-0001-6808-1845

sarroliga@unan.edu.ni

\section{Juan Alberto Betanco}

Doctor en Gestión y calidad de la investigación Científica. Docente Titular (TD) de UNAN-Managua / FAREM-Estelí.

https://orcid.org/0000-0001-8838-8588

betancoja@yahoo.com

\section{RESUMEN}

La implementación de medidas de eficiencia y ahorro energético en edificaciones es fundamental para reducir emisiones de gases de efecto invernadero que tienen impacto en el cambio climático del planeta. En este sentido, las universidades como edificios públicos que usan constantemente la energía, tanto en ocupación, iluminación, climatización y equipos; tienen la responsabilidad de hacer uso eficiente del consumo de ésta. Para medir el comportamiento eficiente, se implementan auditorías energéticas cuyos resultados constituyen bases para la toma de decisiones en la búsqueda por conseguir edificios adecuadamente sostenibles. En este artículo se destaca la eficiencia energética como una tarea que las universidades deben cumplir con responsabilidad. Se abordan conceptos relacionados a la eficiencia energética y se revisan algunos estudios realizados sobre esta temática en universidades de algunos países. La base metodológica de este artículo es la investigación documental bibliográfica y en línea.

(C) 2021 - Revista Científica de FAREM-Estelí.

(c) (i) (2) Este trabajo está licenciado bajo una Licencia Internacional Creative Commons 4.0 Atribución-NoComercial-Compartirlgual.
RECIBIDO

$26 / 03 / 2021$

ACEPTADO 19/052021
PALABRAS CLAVE

Eficiencia energética; sistema de gestión energética; diagnosis energética; auditoría energética. 


\section{ABSTRACT}

The implementation of efficiency and energy savings measure in buildings is essential to reduce greenhouse gas emissions that have an impact on the planet's climate change. In this sense, universities as public building that constantly use energy both in occupancy, lighting, air conditioning and equipment; have the responsibility to make efficient use of energy consumption. In order to measure efficient behavior, energetic audits are implemented, the results of which constitute the basis for decisions making in the quest to achieve adequately sustainable building. This article highlights energy efficiency as a task that universities must fulfill with responsibility. Concepts related to energy efficiency are discussed and some studies carried out on this subject in universities in some countries are reviewed. The methodological basis of this article is the bibliographic and online documentary research.

\section{KEYWORDS}

Energy efficiency; energetic management system; energetic diagnosis; energetic audit. 


\section{INTRODUCCIÓN}

La energía eléctrica juega un papel esencial en actividades para el desarrollo de la sociedad, por ello su consumo debe ser responsable e inteligente. De ahí que es necesario evitar el gasto de la misma en recursos que no son imprescindibles. Se requiere además evaluar dónde y de qué forma se está consumiendo, así como detectar posibles fuentes de derroche. En consecuencia, es necesario tomar medidas que permitan disminuir el costo económico por consumo eléctrico sin afectar productividad y niveles de confort de los usuarios. Adicionalmente reducir la contaminación atmosférica que causa impacto ambiental en el planeta.

Para Leite Franduloso (2017) las preocupaciones acerca de la eficiencia energética en los últimos años han tomado una importancia, no sólo en los aspectos medioambientales, como también económicos. Las iniciativas impulsadas por las directivas nacionales e internacionales, como requisitos de cumplimiento obligatorio, han despertado la responsabilidad medioambiental de las universidades en el ámbito mundial.

En este sentido, en esta búsqueda de ahorro de energía que aporte a la sostenibilidad económica y ambiental, se considera que las universidades juegan un papel relevante como centros de gran consumo de energía eléctrica. Por otra parte, la implementación de medidas de ahorro y eficiencia energética favorece el aumento de la conciencia y el compromiso de la comunidad universitaria en el proceso de gestión de la energía.

Para comprender esta temática, el presente artículo se plantea como objetivo realizar una revisión bibliográfica sobre eficiencia energética y su implementación de edificaciones universitarias. Además, se realiza una revisión sobre estudios de relevancia realizados en universidades de algunos países. La base metodológica de este artículo es la investigación documental bibliográfica y en línea.

La revisión bibliográfica se realiza como parte del proceso de la investigación bajo el tema: Política de eficiencia Energética en la UNAN-Managua para la optimización del consumo de energía. El estudio mencionado se desarrolla como parte del Programa de Doctorado en Gestión y Calidad de la Investigación Científica que imparte la misma universidad.

Este escrito se aborda en dos partes. La primera plantea la revisión teórica sobre los conceptos de eficiencia energética, gestión de la energía y auditorías energéticas. En la segunda parte se hace referencia a estudios de eficiencia energética realizados por investigadores de algunas universidades de los países mencionados. 


\section{MATERIALES Y MÉTODOS}

Para la localización de los documentos bibliográficos se utilizaron diversas fuentes documentales en línea. Se realizó una búsqueda bibliográfica en octubre de 2020 en el Sistema de Bases de Datos de Bibliografía Científica del Programa INASP-PERii. A la vez se realizó búsqueda de información en el repositorio institucional y del Consejo Nacional de Universidades (CNU).

Para la búsqueda se utilizaron los descriptores: eficiencia energética, sistema de gestión energética, diagnosis energética y auditoría energética. Los registros obtenidos oscilaron entre 46 y 67 , tras la combinación de las diferentes palabras clave. También se realizó una búsqueda en internet en el buscador "google académico" con los mismos términos.

Se seleccionaron estudios relevantes que informasen sobre los aspectos de eficiencia energética en edificios universitarios, se realizó la lectura crítica de documentos y de las etapas de auditorías energéticas descritas en los mismos.

\section{RESULTADOS}

La eficiencia energética es la forma más rentable de reducir el consumo de energía. Guerrero Pérez (2016) afirma que la eficiencia energética puede definirse como la disminución del consumo energético, manteniendo los mimos niveles de energía, sin reducir el confort y calidad de vida, cuidando el medio ambiente, garantizando el abastecimiento y fomentando la sostenibilidad en el uso de los mismos.

La eficiencia energética toma en cuenta aspectos relacionados con el consumo energético, como ocupación, iluminación, uso de equipos, aislamiento, calefacción, climatización, agua caliente sanitaria y el uso de fuentes de energías renovables para el abastecimiento energético de los edificios. Para Carretero Peña (2012) la eficiencia energética es la proporción u otra relación cuantitativa entre el resultado en términos de desempeño, de servicios, de bienes o de energía y la entrada de energía.

La implementación de medidas de ahorro y eficiencia energética resulta una necesidad tanto para los edificios existentes como para los que se construyan en los años siguientes, dentro de un escenario de economía sostenible. 
Para Linares Llamas (2009) el ahorro y la eficiencia energética constituyen un elemento fundamental para la mejora del medio ambiente, en especial en lo que se refiere al calentamiento global.

Según informe de la Comisión Europea (2020) la energía es una de las prioridades por tres razones. La primera por la combustión de los combustibles fósiles para producir la energía constituye la fuente antropogénica principal de gases de efecto invernadero y tiene su impacto en el cambio climático. La segunda por el uso continuado y a gran escala de combustibles fósiles no renovables y la necesidad de alcanzar la sostenibilidad. Y finalmente, por la importación de un porcentaje alto y que va en aumento del combustible.

\section{Linares Llamas (2009) acertadamente expresa:}

...el ahorro de energía permite ahorrar nuestros escasos recursos económicos, pospone el agotamiento de nuestros escasos recursos fósiles (de los que sin embargo depende mayoritariamente nuestro suministro energético) $y$, por último, parece revelarse como una de las mejores alternativas para reducir las emisiones de CO2. La clave para la existencia de estos ahorros reside en el hecho de que no consumimos energía, sino servicios energéticos: por tanto, puede ser posible proveer el mismo nivel de servicio energético con un menor nivel de consumo de energía (p.12).

Según Lapido Rodríguez, Gómez Sarduy, \& Monteagudo Yánez (2014) alcanzar mayores niveles de eficiencia energética se pueden seguir dos caminos: uno basado en una mejor administración de la energía con pocas inversiones, implementando un sistema de gestión energética adecuado y otro basado en mejoras tecnológicas, haciendo inversiones en equipos y sistemas más eficientes.

Por otra parte, un sistema de gestión energética define las responsabilidades, los procedimientos, el entrenamiento, la verificación interna, las acciones correctivas y preventivas, y el mejoramiento.

Lapido Rodríguez, et al. (2014) también expresa que un sistema de gestión de energía constituye una estructura documentada que define la política, los objetivos y las responsabilidades de la organización, y establece los procedimientos y procesos de planificación, control, aseguramiento y mejoramiento.

La verificación se realiza a través de la implementación de diagnósticos y auditorías energéticas, a través de las cuales se llevan a cabo mediciones de consumo de superficies para reconocer cómo se distribuyen los consumos energéticos y detectar las oportunidades de mejora. Según García Santander (2020) para implementar de manera adecuada una estrategia de uso eficiente de la energía, es necesario hacer un diagnóstico anticipado que involucre como etapa inicial el levantamiento de información. 
Por su parte Gandía Fornés (2016) manifiesta que un sistema de gestión energética es la parte del sistema de gestión de una organización dedicada a desarrollar e implantar su política energética, así como a gestionar aquellos elementos de sus actividades, productos o servicios que interactúan con el uso de energía (aspectos energéticos).

A continuación, se hace referencia a diferentes conceptualizaciones en cuanto a la definición que se da a una auditoría energética:

Gómez Girini, López \& Fernández (2012) se refiere a la definición de auditorías energéticas de la manera siguiente:

La auditoría energética es una herramienta de diagnóstico y gestión, mediante la aplicación de técnicas que permiten determinar el grado de eficiencia, con la que es utilizada la energía. Consiste el estudio de todas las formas y fuentes de energía por medio de un análsis crítico en una instalación consumidora de energía, para así establecer el punto de partida para la implementación y control de un Programa de ahorro de energía, ya que se determina dónde y cómo es utilizada la misma (pág. 1).

García Sanz-Calcedo, Cuadros, y López Rodríguez (2011) expresan que una auditoría evalúa el nivel de «voracidad» energética de un edificio y aporta un estudio de disminución de costes que posibilite reducir los gastos de explotación, mantenimiento y reposición, contribuyendo a preservar el medio ambiente.

López López \& Figueroa Barrionuevo (2015) indican que una auditoría energética es un estudio de disminución de costos energéticos, abarcando una amplia información, en función del grado con el que se realice el estudio, pudiendo llegar desde un informe básico de propuestas de mejora en equipos que son parte del proceso principal, hasta un estudio completo y detallado de mejoras en la empresa.

Así mismo la Comisión Europea (2020) plantea que cuando se efectúa una auditoría energética, se deben determinar aspectos como: uso y tipo de energía utilizada en la instalación, así como en sus procesos y sistemas integrantes; equipos que utilizan energía, tipo y cantidad de energía utilizada. Además, se deben evaluar las posibilidades de ahorrar energía, garantizar la optimización de aislamiento y optimizar los equipos técnicos, sistemas y procesos asociados.

Por otra parte, se deben valorar las posibilidades de utilizar fuentes alternativas o utilizar energía más eficiente. Al respecto Rey Martínez y Velásco Gómez (2006) manifiestan:

La integración de energías renovables en los edificios está ligado a estrategias políticas que dependen del contexto nacional y de los yacimientos potenciales. Las opciones varían según los países, pero coinciden asociar técnicas consolidadas

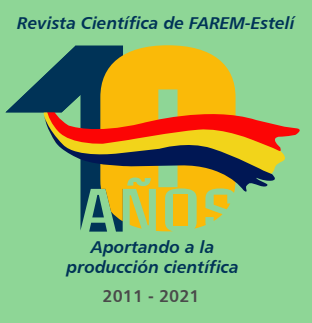


que resultan rentables y competitivas. Algunas alternativas son bombas de calor, colectores solares para agua caliente sanitaria y cogeneración de gas, con tecnologías innovadoras como el uso de células fotovoltáicas y aerogenadores (Pag.25).

European Commission (2018) se refiere al término de fuentes alternativas a cualquier forma de energía diferente a la convencional de generación de energía. La energía convencional puede ser sustituida por fuentes como hidroenergía, cogeneración de gas y otros procesos a partir de los recursos naturales.

Finalmente, Energy (2020) se refiere a que la eficiencia puede permitir el crecimiento económico, reducir las emisiones y mejorar la seguridad energética. Las políticas de eficiencia adecuadas podrían permitir al mundo lograr más del $40 \%$ de los recortes de emisiones necesarios para alcanzar sus objetivos climáticos sin nueva tecnología.

\section{Algunas experiencias de eficiencia energética en edificios universitarios}

García Santander (2020), expone en su estudio que a partir de 2004 la Universidad de Concepción comenzó a levantar información respecto a los edificios y reparticiones existentes al interior del campus, obtuvieron los primeros datos del comportamiento de las instalaciones y consumos de energía eléctrica, generando un diagnóstico centrado en establecer mejoras en las áreas deficientes y obtener reducciones en los montos de facturación y emisiones de $\mathrm{CO}_{2}$.

Descubrieron que el principal consumo de energía en la universidad es la iluminación, la primera implementación fue el recambio tecnológico de ésta en el edificio, y lograron disminuir en un $61 \%$ en la facturación de energía de manera inmediata, además de lograr una mejor calidad y uniformidad en el recinto.

Lo mismo ocurrió con el sistema de alumbrado público del campus, donde con un proyecto de recambio produjo un ahorro de $65 \%$ aproximadamente. Paralelamente, instalaron una plataforma de monitoreo para la gestión eficiente de uso de la energía, identificando estado de fallas, reporte de consumos, entre otras características de gestión.

El estudio realizado por Gaitán Hernández y Pérez (2019) determina la carga térmica de enfriamiento por cada habitación de la residencia estudiantil del recinto Universitario Simón Bolívar (RUSB) de la Universidad Nacional de Ingeniería (UNI), Nicaragua, y propone la instalación de equipos de climatización, para lograr un ambiente de confort higrotérmico en cada una de estas habitaciones. 
El estudio brinda información del edificio, realiza cálculos para determinar la carga térmica de enfriamiento y analiza la selección de equipos tomando en cuenta parámetros de eficiencia energética y la viabilidad de adquisición de los mismos considerando factores económicos y ambientales. También propone un plan de instalación de los equipos y propone plan de mantenimiento de los mismos.

Leite Frandoloso (2017) en su investigación en la de la Universitat Politècnica de Catalunya - UPC, discute diferentes acciones en el planeamiento y en la gestión de los recursos naturales y sus impactos en un área de parque construida en la universidad. Evalúa indicadores para el desempeño energético de los edificios de la universidad y una metodología para la verificación de otro contexto universitario, en este caso, en la Universidade de Passo Fundo - UPF, ubicada en el Sur de Brasil.

En el estudio se definieron los dos escenarios. Temas relevantes al estudio igualmente son abordados de manera paralela: la caracterización de la matriz energética de Brasil y España, el planeamiento y la gestión de los recursos naturales en edificios universitarios; los parámetros normativos para la eficiencia energética y las interrelaciones con el confort térmico de los usuarios.

A partir de estas estrategias se formuló una metodología de abordaje, perfeccionando y ajustando los métodos desarrollados previamente, además de incluir las nuevas pautas generadas por la investigación específica, con el objetivo de contextualizar las características brasileñas y de la UPF. Dicha metodología fue utilizada como punto de referencia para los instrumentos de gestión de los recursos naturales y su repercusión económica en la Universidad.

A partir de los resultados indican pautas para apoyo en los procesos de toma de decisiones para la mejora del desempeño energético, térmico y de confort, es decir contribuyendo con la calidad ambiental de los espacios de aprendizaje y trabajo.

Con este trabajo, observaron los análisis y diagnósticos de la evolución de los factores incidentes en el consumo energético, para que la Universidad promoviera reflexiones en sus prácticas en las actividades académicas y administrativas hacia la puesta en marcha de herramientas que contribuyan para la educación para la sostenibilidad y para el cierre de flujos de materiales y energéticos en sus infraestructuras.

El estudio realizado por López López et al. (2015) realizado en la Facultad de Ingeniería Civil y Mecánica de la Universidad Técnica de Ambato, Ecuador, desarrollaron el análisis energético de las instalaciones con el apoyo de normas técnicas ecuatorianas e internacionales para el control de los niveles 
de eficiencia energética en las instalaciones, calidad del suministro de energía eléctrica y niveles de iluminación.

Para el diagnóstico realizaron inicialmente una inspección visual de las instalaciones para conocer sus condiciones técnico eléctrico y arquitectónico, realizaron levantamiento de cargas instaladas en los edificios y el levantamiento de planos de instalaciones eléctricas de iluminación y tomacorrientes.

Del análisis realizado encontraron oportunidades de mejora para reducir los consumos de energía eléctrica, como la instalación de un banco de capacitores en las acometidas de red para mejorar el factor de potencia del servicio eléctrico, readecuación del espacio físico de la biblioteca, redistribución de los circuitos de iluminación, reemplazo de luminarias e instalación de sensores para encendido de luminaria.

Por su parte Lapido Rodríguez, et al. (2014) en su investigación muestran la necesidad de incrementar la eficiencia energética como una alternativa para lograr un modelo energético sostenible opuesto al paradigma actual.

Plantean la implementación de un sistema de gestión de la energía basado en la norma NC: ISO 50001: 2011, para administrar correctamente los portadores energéticos, alcanzando mejor desempeño y reduciendo el impacto ambiental asociado.

En el estudio describen las fortalezas que existen en Cuba que propician una rápida implementación de estos sistemas de gestión enfatizando la participación de las universidades y de la Red de Energía del Ministerio de Educación Superior.

La investigación realizada por Martínez Flores (2014), estudia el potencial de los sistemas de aporte de luz solar y en particular del sistema SOLATUBE, en cuanto a la capacidad de iluminar espacios al interior de edificios en donde debido a su diseño arquitectónico la luz natural por su naturaleza no puede llegar. La investigación se plantea desde la Universidad de El Salvador.

El documento presenta la metodología documental y los conceptos teóricos fundamentales de la naturaleza de la luz natural, que contribuyan a la comprensión de la propuesta de iluminación con luz natural en el interior de edificios, y en particular utilizando el sistema SOLATUBE.

Describe además la metodología de la simulación, que evalúa la situación de los edificios, desde el punto de vista de ubicación, confort y consumo energético debido a los rubros de iluminación general, climatización y artefactos, a fin de establecer una tendencia predominante de consumo por cada rubro. 
Finalmente presenta los resultados obtenidos a través de la simulación con el software DIALux 4.11, de los proyectos de iluminación utilizando luminarias LED y tubos de luz de la empresa SOLATUBE. El estudio evalúa los proyectos mediante el valor actual neto (VAN) y la tasa interna de retorno (T.I.R), para determinar la opción tecnológica económicamente viable.

\section{CONCLUSIONES}

La presente revisión documental muestra la necesidad de implementar en las universidades un sistema de gestión de la energía como una alternativa para alcanzar un mejor desempeño y reducir el impacto ambiental asociado. La necesidad de disminuir los consumos energéticos para reducir el impacto ambiental ocupa un lugar relevante en la actualidad, y en ello se justifica la necesidad de establecer e implementar programas de gestión energética en general.

La participación de las universidades en la implementación de este tipo de programas enfocados en la eficiencia energética, que tienen como objetivo común alcanzar el óptimo uso de energías que además de beneficiar en lo económico, pueden crear protección, cuidado y responsabilidad medioambiental. Por otra parte, brindan la oportunidad de incorporar nuevas fuentes energéticas a base de recursos naturales disponibles en el territorio. Al analizar estudios realizados por otras universidades, se observa que la obtención de resultados mediante la implementación de auditorías energéticas en edificios universitarios, posibilita determinar las características energéticas de éstos y permite obtener analizar opciones para la mejora de dichas características energéticas.

Por otra parte, facilita la toma de decisiones para invertir en mejoras del sistema de gestión energética y en la implementación de fuentes renovables en los campus, ampliando la investigación en esta área de estudios y similares.

Los estudios que se han realizado en distintas instituciones académicas muestran factores importantes para el cuestionamiento de la calidad de los edificios, de su demanda energética, características de la gestión de la energía. Estos estudios permiten identificar potenciales de ahorro, proponer ajustes para la eficiencia energética.

Finalmente se puede afirmar que las investigaciones sobre eficiencia energéticas en las universidades son procesos necesarios para aplicar criterios que permitan la mejora tanto de edificios existentes como en los nuevos, en relación al consumo de energía y en la reducción de la contaminación por emisiones de $\mathrm{CO}_{2}$. Estos estudios permiten valorar factores incidentes en el 
consumo de energía y las condiciones de confort térmico, que contribuyen a la toma de decisiones acertadas.

\section{BIBLIOGRAFÍA}

Carretero Peña, A. (2012). Gestión de la eficiencia energética: cálculo del consumo, indicaciones y mejora. AENOR - Asociación Española de Normalización y Certificación. Recuperado el 26 de septiembre de 2020, de https://elibro.net/es/ereader/unanmanagua/53576? page $=49$

Comisión Europea. (10 de Octubre de 2020). European Commission. Obtenido de http://eippcb.jrc.es

Energy, G. C. (05 de julio de 2020). Green Corporate Energy. Obtenido de Energy Efficiency: https://greencorporatenergy.com/es/eficienciaenergetica-para-mejorar-el-futuro/

European Commission. (2018). JRC Science for policy report. Fossil CO2 emissions of all world countries. Luxembourg: Publications Office of the European Union. doi:doi:10.2760/30158

Gaitán Hernández, C. E., \& Pérez, K. S. (2019). Universidad Nacional de Ingeniería. Obtenido de Diseño de un sistema de climatización para la residencia estudiantil del Recinto Universitario Simón Bolívar de la Universidad Nacional de Ingeniería : http://ribuni.uni.edu.ni/3435/

Gandía Fornés, J. (2016). Universitat Jaume I. Recuperado el 16 de Agosto de 2020, de Módulo I. Sistemas de gestión de energía: http://hdl.handle. net/10234/171233

García Santander, L. (4 de Junio de 2020). Universidad de Concepción. Recuperado el 12 de octubre de 2020, de Eficiencia Energética: http:// www.iit.udec.cl/eficiencia-energetica-como-la-via-para-una-vidasustentable/

García Sanz-Calcedo, J., Cuadros, F., \& López Rodríguez , F. (2011). La auditoría energética: una herramienta de gestión en atención primaria. Scielo, 25(6), 3. Recuperado el 10 de 10 de 2020, de https://www. scielosp.org/article/gs/2011.v25n6/549-551/

Gómez Girini, R., López , G. R., \& Fernández, J. F. (2012). Metodologías para auditorías energéticas en edificios. IRESE, 17. Recuperado el 10 de 10 de 2020, de http://www.academia.edu/download/48772831/09metodologias-_para_auditorias_energeticas.pdf

GuerreroPérez, R. (2016). Fundamentos delaedificaciónyeficiencia energética. Málaga, España: IC Editorial. Recuperado el 25 de Septiembre de 2020, de https://elibro.net/es/ereader/unanmanagua/43901 ?page $=10$

Lapido Rodríguez, M., Gómez Sarduy, J. R., \& Monteagudo Yánez, J. P. (2014). Participación de la universidad en la mejora de la eficiencia energética del sector productivo cubano. Universidad y Sociedad, 8. Obtenido de http://rus.ucf.edu.cu/ 
Leite Frandoloso, M. A. (Septiembre de 2017). Tesis Doctorals en Xarxa. doi: http://hdl.handle.net/10803/461416

Linares Llamas, P. (Marzo - Abril de 2009). Eficiencia energética y medio ambiente. Economía y Medio Ambiente ICE(847). Recuperado el Febrero de 2020, de https://www.researchgate.net/publication/28312979 Eficiencia_energetica_y_medio_ambiente

López López, G. A., \& Figueroa Barrionuevo, E. A. (2015). Universidad Técnica de Ambato. Obtenido de Auditoría energética de los edificios administrativo y docente de la Facultad de Ingeniería Civil y Mecánica de la Universidad Técnica de Ambato para disminuir el consumo de energía eléctrica: http://192.188.46.193/handle/123456789/12380

Martínez Flores, M. E. (2014). UNAN León. Obtenido de Propuesta de ahorro energético mediante sistemas de aporte de luz natural en los edificios del campus central de la universidad de El Salvador: http://repositorio. cnu.edu.ni/Record/RepoUNANL4295

Rey Martínez, F. J., \& Velásco Gómez, E. (2006). Eficiencia energética en edificios. Certificación y auditorías energéticas. Madrid, España: Thomsom Editores Espain. Recuperado el 13 de 10 de 2020, de shorturl.at/dfAUX

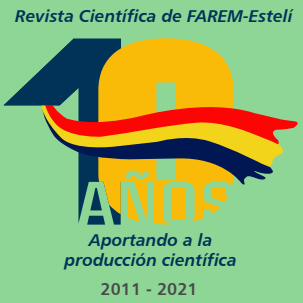

